\title{
Collaborative Development of Outing Assistants for People with Dementia: a Case Study on a Co-design Approach
}

\author{
Atsushi Omata \\ Shizuoka University \\ Hamamatsu, Shizuoka, Japan \\ omata@kirilab.net \\ Mia Kobayashi \\ Shizuoka University \\ Hamamatsu, Shizuoka, Japan
}

\author{
Shogo Ishikawa \\ Shizuoka University \\ Hamamatsu, Shizuoka, Japan \\ Shinya Kiriyama \\ Shizuoka University \\ Hamamatsu, Shizuoka, Japan
}

\begin{abstract}
People with dementia (PwD) have been conducting educational activities to live well with dementia. Appropriate CHI design is needed to support the lives of $\mathrm{PwD}$. However, current prototypical systems are mostly designed for needs on the surface and do not approach hidden needs. To develop an system that PwD wants to use on a daily basis, we conducted a co-production practice with $\mathrm{PwD}$ focussing on outing their home. We collaborate with a group of early-onset dementia who wanted their opinions to be reflected in ICT. A participatory design workshop was undertaken to discuss the development of outing assistants. As a result, it was found that they did not want the system to be responsible for intervening in their disabilities, but to positively encourage their activities. To conduct a case study for outing assists, we focused on a person with dementia's activity to verify whether it contributes to her life. The system was implemented as a voice and visual interaction system, designed to encourage a positive relationship between the supporter and her. Our results indicate that the system design to support PwD should produce positive interaction and should be adapted to the individual context.
\end{abstract}

\section{CCS CONCEPTS}

- Human-centered computing $\rightarrow$ User centered design.

\section{KEYWORDS}

co-design, dementia, speech dialogue system

\section{ACM Reference Format:}

Atsushi Omata, Shogo Ishikawa, Mia Kobayashi, and Shinya Kiriyama. 2021. Collaborative Development of Outing Assistants for People with Dementia: a Case Study on a Co-design Approach. In Asian CHI Symposium 2021 (Asian CHI Symposium 2021), May 8-13, 2021, Yokohama, Japan. ACM, New York, NY, USA, 4 pages. https://doi.org/10.1145/3429360.3468173

Permission to make digital or hard copies of part or all of this work for personal or classroom use is granted without fee provided that copies are not made or distributed for profit or commercial advantage and that copies bear this notice and the full citation on the first page. Copyrights for third-party components of this work must be honored. For all other uses, contact the owner/author(s).

Asian CHI Symposium 2021, May 8-13, 2021, Yokohama, Japan

(C) 2021 Copyright held by the owner/author(s).

ACM ISBN 978-1-4503-8203-8/21/05.

https://doi.org/10.1145/3429360.3468173

\section{INTRODUCTION}

Dementia is defined as a syndrome in which there is deterioration in memory, thinking, behavior, and the ability to perform everyday activities. The decline in cognitive function makes it difficult to do things that used to be taken for granted, such as dressing, eating, and excretion. However, they are able to continue to live at the same level as before by removing frictions such as uneasiness and distastefulness through environmental design [16]. We use the environment in an inclusive sense, including people and things around us. From the perspective of environmental design, there are two approaches to dementia support.

- Interaction support between a PwD and an environment

- Interaction support between a PwD and a caregiver

In the first point, the Dementia Services Development Centre at the University of Stirling organized the evidence in a systematic review of the architectural design features required for living environments for people with dementia and presented guidelines for dementiafriendly design [2]. On the other point, the communication skills approach enhances the quality of life (QOL) of PwD and increases positive interactions in various care settings [5]. Both approaches lead to keep QOL of Pwd. Also, recent studies [3] have shown that it is important for PwD to have a purpose in life to improve their sustainable QOL. However, because of the limitations of going it alone, many $\mathrm{PwD}$ loses their purpose in life. A purpose in life cannot be found in their absence. Therefore, an important perspective is to collaborate with $\mathrm{PwD}$ to develop systems for empowering them to do what they want to do and building a better relationship with them. In this paper, we describe the results of the development of an outing assistant in collaborative work with PwD.

\section{RELATED WORK}

Many studies have been conducted on the use of ICT to support PwD, including studies on monitoring systems [12] [18], studies on assisting memory and judgment abilities [15] [19], and studies on mental support [8] [4]. These studies are aimed at complementing the functions impaired by cognitive disorders or reducing the burden on caregivers. However, it has been pointed out that it is difficult for people with disabilities to accept such a system because it emphasizes their weaknesses [14]. Traditional approaches are reaching their limits in terms of how the systems designed by an engineer contribute to the quality of life of people with disabilities. 
In recent years, the concept of "co-production", in which PwD participates in research to create a new value, has become widespread [10] [9] [11]. Holbo et al. performed co-design workshops with early-stage dementia and their family caregivers to identified several factors that influence people with dementia's attitudes toward safe walking technology, and how they want the technology to assist them [7]. Bourazeri et al. proposed the PERCEPT approach for the co-design process and develop a smart home toolset for a person living with early-stage dementia or Parkinson's [1]. Our case study aims to explore the features required in spoken dialogue systems used by people with early-onset dementia ${ }^{1}$, and to present a system to improve their quality of life.

\section{CO-DESIGN OF OUTING ASSISTANTS}

Our case study has been carried out in 3 steps. Step 1) Organize a workshop to identify issues in spoken dialogue systems. Step 2) Implement a prototype based on the extracted issues. Step 3) Get feedback on our proposed system from PwD.

\subsection{Workshop}

PwD faces various barriers to going out, such as difficulty in changing clothes, not knowing where to go, and estimating the time to go out. Therefore, we focused on the support for outings, which is the first barrier to the realization of what the PwD wants to do. First of all, we created a scenario of the outing assistants(Table 1) and held a workshop to get opinions from PwD. The workshop was organized as part of the monthly exchange meeting for Ayumi-no-Kai ${ }^{2}$. The participants of the workshop were members of Ayumi-no-Kai. The workshop was conducted in the form of focus group interviews, in which the participants were asked about their opinions on the scenario and their usual routines before going out. Seven $\mathrm{PwD}$, one caregiver, and one researcher participated in the workshop, and the researcher and caregiver served as facilitators. The age of the PwD participants was 50-60s. Three were males and four were females. And the PwD participants was able to express themselves verbally. The following is a list of comments received on the scenarios in Table 1.

- Too much detail can be confusing.

- I don't like to hear the same words over and over again.

- I don't like "not yet" and other urgent phrases like "not yet" because it makes me nervous.

- Praise motivates me.

- I'd rather have your cheer than confirmation.

- I'd appreciate it if you'd check the locks.

- It's good to have a relatable character.

- It might be easier to understand if there was a display like a list of things to do on the display.

The experience of dementia is different, depending on the cause [17], life experiences [6], and the stage of dementia [13]. Although there was a variety of opinions among the participants, they all agree that "positively encourage our activities" is a common factor for HCI design.

\footnotetext{
${ }^{1}$ Early-onset dementia is defined as the onset of symptoms before the age of 65 years ${ }^{2}$ Ayumi-no-Kai is a group of people with early-onset dementiaand their families.
}

Table 1: Scenarios for the first version of an outing assistant.

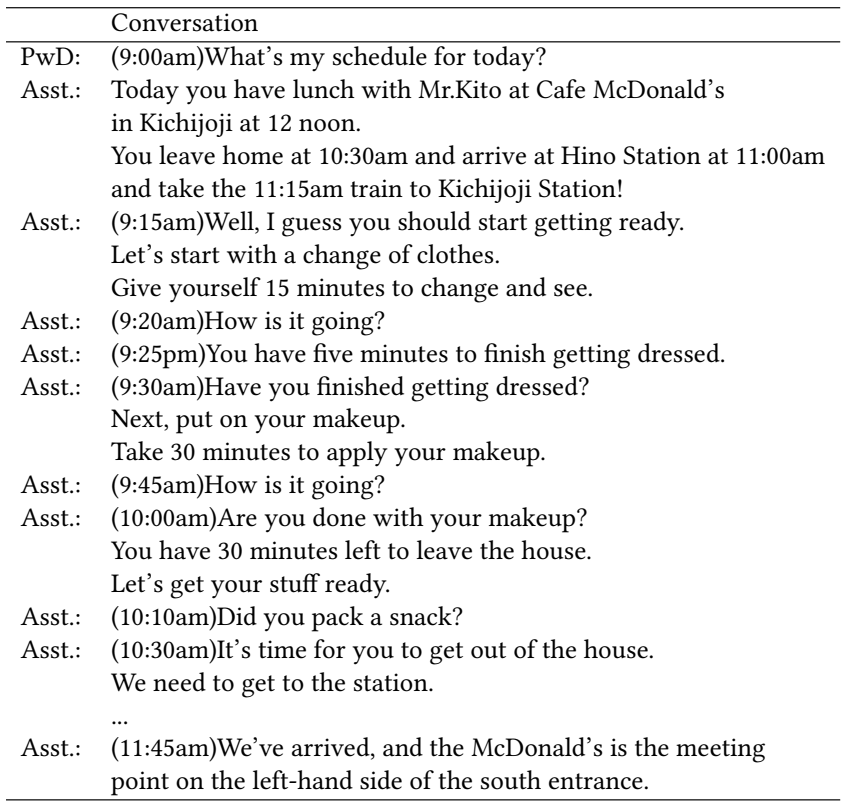

\subsection{System design}

Based on the feedback we received, we designed a system to support a 60-year-old woman with Alzheimer's disease who participated in the workshop. She has been giving lectures on her experiences as a PwD, and we selected her for this study so that we could gather information on her specific symptoms. The following items were studied in detail and applied to the design of the system.

- What the person can and cannot do.

- What steps are involved in getting up and going out in the morning?

- What kind of problems does the person have?

- What kind of support does the family provide for the person's problems?

- What is the gap between what the person is having difficulties with and what the family is having difficulties with?

- Could a system provide the support that would make it easier for me to not have to worry about my family and caregivers?

- How can we work with systems as much as possible to fulfill the person's wishes?

Her difficulties in her daily life are described below. Because she needs assistance with many of her daily activities due to her impaired visuospatial ability, she is usually assisted by a caregiver to prepare for her outings. She mentioned that she tended to choose clothes that were easier to wear than the ones she wanted to wear. She also mentioned that the makeup would look different depending on who was helping her with it. As shown above, she has difficulty communicating her intentions to her caregivers because she cares so much about her caregivers. If a system is able to play a role as an intermediary between a person with dementia and her caregiver, it will encourage the motivation to go out. Therefore, we propose that the system of communication between a person living with 


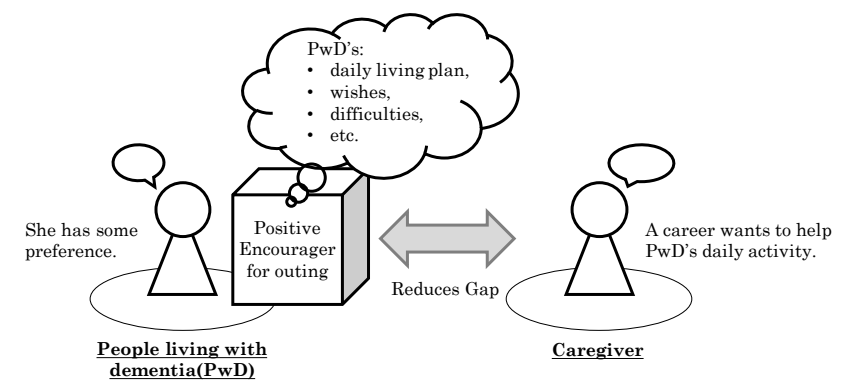

Figure 1: Our proposal for interaction with Positive Encourager.

Table 2: A conversation asking about schedules.

\begin{tabular}{ll}
\hline Conversation \\
\hline PwD: & What's my schedule for today? \\
Asst.: & You're going to go to an eel restaurant with your friends today! \\
& The carefully selected eel is characterized by its moderate fat, \\
& richly tasted meat, and the fragrant smell of the burned skin. \\
\hline
\end{tabular}

dementia and caregivers, as shown in Figure 1, will enable the practice of caring for $\mathrm{PwD}$ that reflects their wishes and difficulties.

\subsection{Implementation of Prototype}

The target user uses a speech dialogue system (Siri) in her daily life, so we implemented a voice and visual-based outing assistant system. The development of a voice and visual interaction system using Google Nest $\mathrm{Hub}^{3}$ with smart speakers. The Google Nest Hub is equipped with a display, so it is possible to deliver visual as well as audio data to users. The processing of the voice interaction between the user and the system was implemented using Dialogflow ${ }^{4}$, a platform for creating conversational interfaces. We developed a scenario in which a system intervenes in the situations of the problems of PwD and their caregivers, which were extracted in the previous section. For the scenarios, we selected scenes that can be supported by spoken dialogue systems in the process of the target user's outing. Specifically, we created scenarios in three scenes: schedule confirmation, makeup suggestions, and clothing suggestions. In addition, scenarios were created using the opinions extracted from the workshop in the previous section. Table 2, 3 , and 4 show how the spoken dialogue systems and the user interact with each other. By displaying pre-registered images of clothing and makeup, PwD can think together with their caregivers based on the suggestions.

\subsection{Feedback}

We asked one PwD who was the target of the study and one of her caregivers about their impressions of our proposed system. We received positive comments from the $\mathrm{PwD}$, such as "I sometimes hesitate to wear clothes that I want to wear because it is difficult to do so, but this system makes it easy to ask caregivers to suggest clothes that I want to wear. We also received positive comments

\footnotetext{
${ }^{3}$ https://store.google.com/product/google_nest_hub

${ }^{4} \mathrm{https}: / /$ dialogflow.com
}

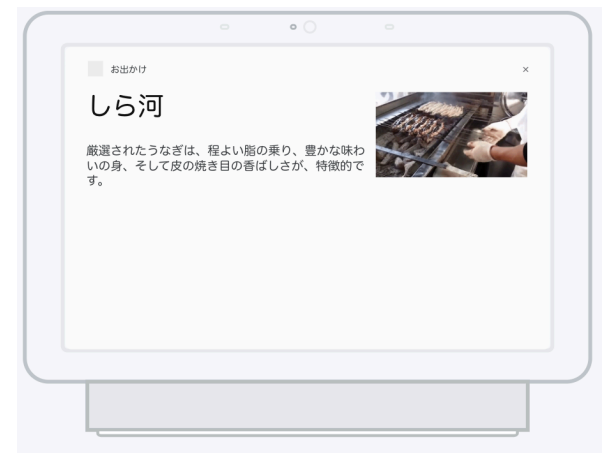

Figure 2: Screen output for confirmation of the schedule.

Table 3: A conversation about makeup recommendation.

\begin{tabular}{ll}
\hline & Conversation \\
\hline PwD: & What am I going to do with my makeup today? \\
Asst.: & Today, You're going to eat a delicious eel with your friends, \\
& so I recommend you to wear a natural makeup. \\
Caregiver: & How about some pretty makeup? \\
PwD: & I'd like to wear some pretty makeup. \\
Asst.: & This is the pretty makeup. \\
Caregiver: & Which one do you prefer? \\
PwD: & The natural one. \\
\hline
\end{tabular}

Table 4: A conversation that suggest clothing recommendations.

\begin{tabular}{ll}
\hline & Conversation \\
\hline PwD: & What should I wear today? \\
Asst.: & Today it's up to $35^{\circ} \mathrm{C}$ and $60 \%$ humidity, so cool clothes \\
& are recommended. It is a candidate. \\
Caregiver: & Which one do you want? \\
PwD: & I'll take this one. \\
\hline
\end{tabular}

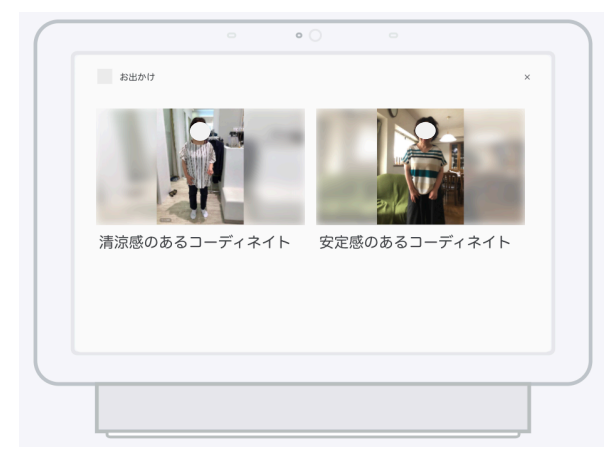

Figure 3: Screen output for clothing recommendations.

from the caregiver, such as "I want her to wear the clothes she wants to wear, but sometimes I don't know what she wants to wear, so the system has helped me to think about it with her." It is suggested that this will lead to the realization of support to realize what the person wants to do while maintaining the relationship between PwD and caregivers. 


\section{DISCUSSION AND CONCLUSION}

In this paper, we have examined the requirements for a spoken dialogue system for PwD using a co-design approach. From the co-design workshop, we found that they did not want the spoken dialogue system to be responsible for intervening in their disabilities, but to positively encourage their activities. These findings indicate that in people with early-onset dementia, it is important to support them to achieve what they want to do, rather than to intervene in disabilities, which has been the focus of many HCI studies. We also have developed a prototype of an outing assistant focused on supporting the activities of a person with dementia. The system was implemented as a voice and visual interaction system for three situations: schedule confirmation, makeup suggestions, and clothing suggestions. Our results indicate that the system design to support PwD should produce positive interaction and should be adapted to the individual context. However, there are limitations in adapting the scenario to other people because it was created for one PwD. By creating and generalizing scenarios for a variety of symptoms, it will be possible to provide support with a wider range of applicability. The participation of PwD is essential for the development of dementia-friendly technologies, and the realization of dementia-friendly technologies will lead to the realization of everyone-friendly technologies.

\section{ACKNOWLEDGMENTS}

We are extremely grateful to members of Ayumi-no-Kai who willingly participated in the study and provided invaluable data.

\section{REFERENCES}

[1] Aikaterini Bourazeri and Simone Stumpf. 2018. Co-designing smart home technology with people with dementia or Parkinson's disease. In Proceedings of the 10th Nordic Conference on Human-Computer Interaction. 609-621.

[2] Alison Bowes and Alison Dawson. 2019. Designing Environments for People with Dementia. Emerald Publishing Limited.

[3] Patricia A Boyle, Aron S Buchman, Robert S Wilson, Lei Yu, Julie A Schneider and David A Bennett. 2012. Effect of purpose in life on the relation between Alzheimer disease pathologic changes on cognitive function in advanced age. Archives of general psychiatry 69, 5 (2012), 499-504

[4] Joost Broekens, Marcel Heerink, Henk Rosendal, et al. 2009. Assistive social robots in elderly care: a review. Gerontechnology 8, 2 (2009), 94-103.

[5] C Delmas. 2013. Are difficulties in caring for patients with Alzheimer's disease becoming an opportunity to prescribe well-being with the Gineste-Marescotti® care methodology. IPRIM Institut pour la recherche et l'information sur la méthodologie de soin Gineste-Marescotti®. France. Recuperado de http://www. igm-formation. net/images/stories/pdf/poster_iprim_anglais_2013-eugms. pdf (2013).

[6] Marc A Garcia, Brian Downer, Chi-Tsun Chiu, Joseph L Saenz, Kasim Ortiz, and Rebeca Wong. 2021. Educational benefits and cognitive health life expectancies: Racial/ethnic, nativity, and gender disparities. The Gerontologist 61, 3 (2021), 330-340.

[7] Kristine Holbø, Silje Bøthun, and Yngve Dahl. 2013. Safe walking technology for people with dementia: what do they want?. In Proceedings of the 15th International ACM SIGACCESS Conference on Computers and Accessibility. 1-8.

[8] Stephen C Kramer, Erika Friedmann, and Penny L Bernstein. 2009. Comparison of the effect of human interaction, animal-assisted therapy, and AIBO-assisted therapy on long-term care residents with dementia. Anthrozoös 22, 1 (2009), $43-57$.

[9] Amanda Lazar, Austin L Toombs, Kellie Morrissey, Gail Kenning, Jennifer Boger and Rens Brankaert. 2018. HCIxDementia workshop: engaging people living with dementia. In Extended Abstracts of the 2018 CHI Conference on Human Factors in Computing Systems. 1-7.

[10] Cristian Leorin, Eloisa Stella, Christopher Nugent, Ian Cleland, and Cristiano Paggetti. 2019. The value of including people with dementia in the co-design of personalized eHealth technologies. Dementia and geriatric cognitive disorders 47 , 3 (2019), 164-175.

[11] Stephen Lindsay, Katie Brittain, Daniel Jackson, Cassim Ladha, Karim Ladha, and Patrick Olivier. 2012. Empathy, participatory design and people with dementia.
In Proceedings of the SIGCHI Conference on Human Factors in Computing Systems. 521-530.

[12] Ahmad Lotfi, Caroline Langensiepen, Sawsan M Mahmoud, and Mohammad Javad Akhlaghinia. 2012. Smart homes for the elderly dementia sufferers: identification and prediction of abnormal behaviour. Fournal of ambient intelligence and humanized computing 3, 3 (2012), 205-218.

[13] Susan L Mitchell, Joan M Teno, Dan K Kiely, Michele L Shaffer, Richard N Jones, Holly G Prigerson, Ladislav Volicer, Jane L Givens, and Mary Beth Hamel. 2009. The clinical course of advanced dementia. New England fournal of Medicine 361, 16 (2009), 1529-1538.

[14] Donald A. Norman. 2013. The Design of Everyday Things. Basic Books.

[15] Sonja A O’Neill, Sarah Mason, Guido Parente, Mark P Donnelly, Christopher D Nugent, Sally McClean, Bryan Scotney, and David Craig. 2011. Video reminders as cognitive prosthetics for people with dementia. Ageing International 36, 2 (2011), 267-282.

[16] Alison Phinney, Habib Chaudhury, and Deborah L O'connor. 2007. Doing as much as I can do: The meaning of activity for people with dementia. Aging and Mental Health 11, 4 (2007), 384-393.

[17] Limor Raz, Janice Knoefel, and Kiran Bhaskar. 2016. The neuropathology and cerebrovascular mechanisms of dementia. Fournal of Cerebral Blood Flow \& Metabolism 36, 1 (2016), 172-186.

[18] Lin Wan, Claudia Müller, Volker Wulf, and David William Randall. 2014. Addressing the subtleties in dementia care: pre-study \& evaluation of a GPS monitoring system. In Proceedings of the SIGCHI conference on human factors in computing systems. 3987-3996.

[19] Maria Klara Wolters, Fiona Kelly, and Jonathan Kilgour. 2016. Designing a spoken dialogue interface to an intelligent cognitive assistant for people with dementia. Health informatics journal 22, 4 (2016), 854-866. 